\title{
A Monolithic MQW InP-InGaAsP-Based Optical Comb Generator
}

\author{
Cyril C. Renaud, Marianna Pantouvaki, Sylvie Grégoire, Ian Lealman, Member, IEEE, Paul Cannard, \\ Simon Cole, Ron Moore, Russell Gwilliam, and Alwyn J. Seeds, Fellow, IEEE
}

\begin{abstract}
We report the first demonstration of a monolithic optical-frequency comb generator. The device is based on multisection quaternary/quaternary eight-quantum-well InP-InGaAsP material in a frequency-modulated (FM) laser design. The modulation is generated using quantum-confined Stark-effect phase-induced refractive index modulation to achieve fast modulation up to $24.4 \mathrm{GHz}$. The laser was fabricated using a single epitaxial growth step and quantum-well intermixing to realize low-loss phase adjustment and modulation sections. The output was quasicontinuous wave with intensity modulation at less than $20 \%$ for a total output power of $2 \mathrm{~mW}$. The linewidth of each line was limited by the linewidth of the free running laser at an optimum of $25 \mathrm{MHz}$ full-width at half-maximum. The comb generator produces a number of lines with a spacing exactly equal to the modulation frequency (or a multiple of it), differential phase noise between adjacent lines of $-82 \mathrm{dBc} / \mathrm{Hz}$ at $1-\mathrm{kHz}$ offset (modulation source-limited), and a potential comb spectrum width of up to $2 \mathrm{THz}(15 \mathrm{~nm})$, though the comb spectrum was not continuous across the full span.
\end{abstract}

Index Terms-Frequency-modulated (FM) laser, laser diode, optical frequency comb generation.

\section{INTRODUCTION}

$\mathbf{M}$ ANY different applications, ranging from dense wavelength-division-multiplexed (WDM) optical communications [1] to photonic $\mathrm{THz}$ synthesis [2]-[4], require a reliable and cost-effective frequency reference source. The most reliable sources as frequency reference are atomic or molecular resonances [5], [6]. They also offer a large number of frequency lines in a relatively dense grid [7], [8], However, they are not suitable for integrated systems. Several other solutions have been proposed to provide a regularly spaced frequency comb, such as

Manuscript received December 22, 2006; revised April 24, 2007. This work was supported by the U.K. Engineering and Physical Science Research Council (EPSRC) and by the European Commission.

C. C. Renaud and A. J. Seeds are with the Department of Electronic and Electrical Engineering, University College London, London WC1E 7JE, U.K. (e-mail: c.renaud@ee.ucl.ac.uk; crenaud@ucl.ac.uk; a.seeds@ee.ucl.ac.uk; aseeds@ucl.ac.uk).

M. Pantouvaki was with the Department of Electronic and Electrical Engineering, University College London, London, WC1E 7JE U.K. She is now with IMEC, B-3001 Leuven, Belgium (e-mail: m.pantouvaki@ee.ucl.ac.uk; mpantouvaki@ucl.ac.uk).

S. Grégoire was with the Department of Electronic and Electrical Engineering, University College London, London WC1E 7JE, U.K. She is now with the École National Superieure de Telecommunication, CS 83818-29238 Brest Cedex, France (e-mail: s.gregoire@ee.ucl.ac.uk).

I. Lealman, P. Cannard, S. Cole, and R. Moore are with the Centre for Integrated Photonics, Ipswich IP5 3RE, U.K. (e-mail: ian.lealman@ ciphotonics.com; paul.cannard@ciphotonics.com; simon.cole@ciphotonics. com; ron.moore@ciphotonics.com).

R. Gwilliam is with the Ion Beam Centre, University of Surrey, Guildford GU2 7RX, U.K. (e-mail: r.gwilliam@surrey.ac.uk).

Digital Object Identifier 10.1109/JQE.2007.904522 a Fabry-Perot interferometer [9] or a fiber ring resonator [10], both of which can offer a large frequency comb span. The frequency accuracy and stability of these devices compared with the atomic or molecular resonances are limited by the optical length of the resonators and their mechanical stability (sensitivity to vibration and thermal changes), though they could be stabilized by locking them on an atomic or molecular transition. Another solution is the use of deep angle modulation of an optical source to generate precisely spaced frequency lines [11], [12]. Such systems can be reasonably compact and provide lines over a 4-THz ( $-50-\mathrm{dB}$ bandwidth) span [12]. However, the power of each line is small (from $10 \mu \mathrm{W} /$ line at the seed laser peak down to $1 \mathrm{nW} /$ line $3 \mathrm{THz}$ from the peak) and the number of frequency lines and their spacing are limited by the difficulty of realizing such modulation at high frequency. Ultra wide frequency comb generators ( $30 \mathrm{THz}$ ) have also been demonstrated by further increasing the comb width using self-phase modulation in an optical fiber [13]. Other solutions such as an amplified fiber loop comb generator [14], [15] or mode-locked semiconductor lasers [16]-[18] can offer hundreds of lines with a spacing from 10 to $25 \mathrm{GHz}$ over a band of at least $1 \mathrm{THz}$ with a stability limited by the thermal stability of the semiconductor laser (master laser in the case of the fiber loop).

The principle of the FM laser [19] is an extension of the FM mode-locked laser solution and should offer a large number of equally spaced frequency lines, spaced by the modulation source frequency, over the gain spectrum of the semiconductor laser, but without the strong intensity-modulated envelope and the critical frequency tuning of a mode-locked laser which is detrimental to some filtering schemes. In this paper, we describe such a monolithic InP-InGaAsP FM laser optical comb generator using the quantum-confined Stark effect (QCSE) [20] as the refractive index modulation mechanism. The laser described in this paper provides a few comb lines spaced by $24.4 \mathrm{GHz}$ over a noncontinuous $15-\mathrm{nm}$ wavelength $(2 \mathrm{THz})$ span. It shows stable performance and an almost constant output power of up to $2 \mathrm{~mW}$ (an average of $20 \mu \mathrm{W} /$ line, as the number of lines is limited). Furthermore, the phase noise generated when heterodyning two successive lines was limited by the phase noise of the modulation source used, and the linewidth of each line created was equal to the free-running laser linewidth. The different issues related to the fabrication of this laser and some of the operation limitations will also be discussed.

\section{PRINCIPLE OF THE FM SOURCE}

FM laser operation occurs when the laser cavity phase is modulated at a frequency close to the axial frequency [19]. In ideal 


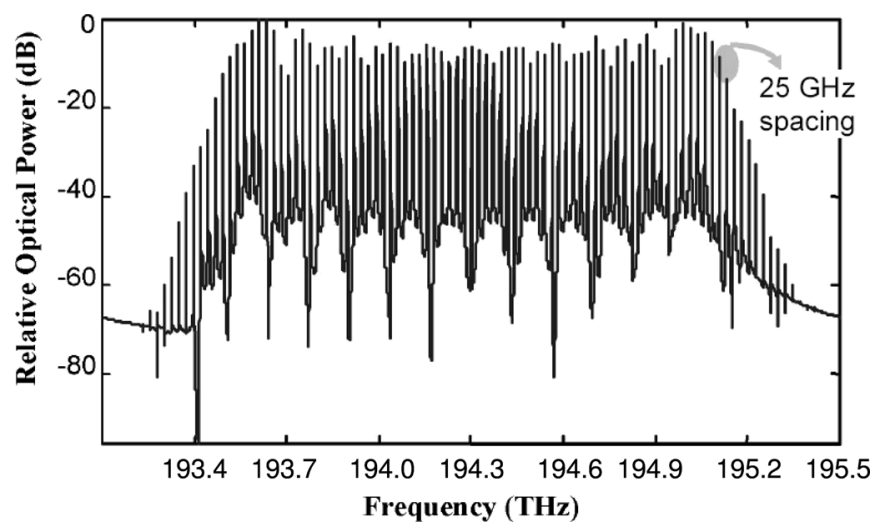

Fig. 1. Theoretical spectrum of an FM laser.

FM laser operation, there is frequency modulation and no intensity modulation, and the spectrum will comprise a number of lines spaced by the modulation frequency depending on the frequency detuning from the axial mode and the amplitude of the modulation. When the modulation frequency is equal to the axial frequency, the laser will transfer to a mode-locked pulse regime [21]. The FM laser regime is generated by the phase modulation which induces coupling between the different modes of the cavity. The resulting spectrum can be described by the following equations:

$$
\begin{aligned}
E(t) & =E_{0} \sum_{n=-\infty}^{\infty} J_{n}(\Gamma) e^{j\left(\omega_{0}+n \omega_{m}\right) t} \\
\Gamma & =\frac{\Delta \Phi}{2 \pi} \frac{\omega_{\mathrm{ax}}}{\left|\omega_{\mathrm{ax}}-\omega_{m}\right|} .
\end{aligned}
$$

In these equations, $E_{0}$ is the amplitude of the optical field, $\omega_{0}$ is the laser operating frequency, $\omega_{\mathrm{m}}$ is the modulation frequency, $\omega_{\mathrm{ax}}$ is the axial mode spacing of the Fabry-Perot cavity, $\Delta \Phi$ is the amplitude of the phase modulation, and $J_{\mathrm{n}}$ is an ordinary Bessel function. Note that these equations represent an ideal laser with a flat gain spectrum and pure single-mode operation (the fundamental mode is described as a Dirac function). This perfect theoretical laser will generate a spectrum as shown in Fig. 1. To represent a more realistic laser, one can introduce a Lorentzian function to describe the laser operating-mode linewidth and simulate what will happen if this laser has several modes operating at the same time (free running) by summing the FM spectra of each mode. Considering the approximations mentioned above, (1) can be rewritten as

$$
\begin{aligned}
E(t)= & \sum_{l=0}^{K} E_{0} \sum_{n=-\infty}^{\infty} L_{w}\left(\omega-\left(\omega_{l}+n \omega_{m}\right)\right) \\
& \times J_{n}(\Gamma) \cdot \ldots e^{j\left(\omega_{l}+n \omega_{m}\right) t}
\end{aligned}
$$

where $L_{w}(\omega)$ is a Lorentzian function where the mode linewidth is $w$, and we have a total number of $K$ modes operating in the laser cavity. Note that (2) remains the same. Fig. 2 shows a selection of lines created by the FM modulation when three modes are operating in the cavity. As expected, the lines are broader, as they are a superimposition of the different FM created lines from each of the free-running modes. Furthermore, as the spacing of

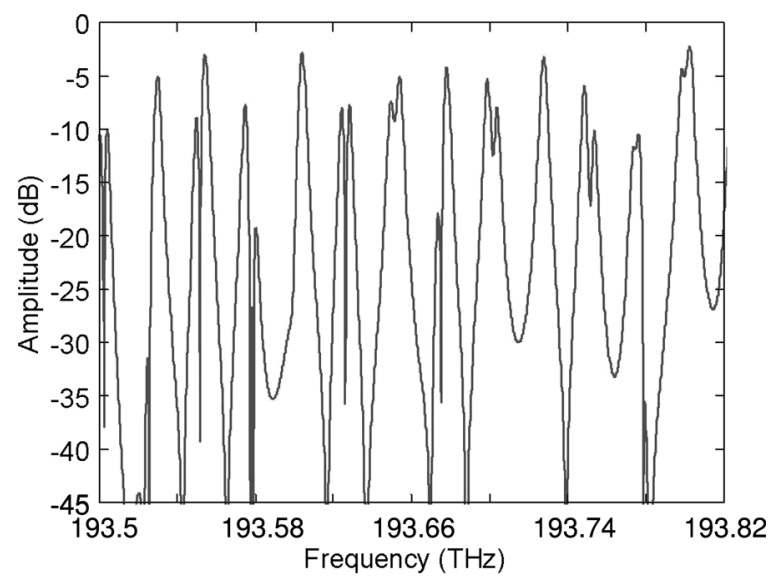

Fig. 2. Theoretical comb spectrum generated by a multimode laser.

the FM comb and the spacing of the free-running laser modes are slightly different, the linewidth of each resulting comb line will depend on its distance from the free-running laser modes. Therefore, for optimum operation, the FM laser should exhibit single-mode operation in the free-running mode (i.e., when not modulated).

\section{DESCRIPTION OF THE LASER}

Fig. 3(a) shows a schematic of the three-section laser. The laser was $1.85 \mathrm{~mm}$ long ( $25 \mathrm{GHz}$ axial mode spacing) with $170-\mu \mathrm{m}$-long modulation and phase sections. The phase section was used to change the axial mode spacing and thus the frequency detuning while the modulation frequency could remain constant. Fig. 3(b) shows the epitaxial structure of the material used for the laser. The structure was grown by metal-organic vapor phase epitaxy (MOVPE) on a semi-insulating InP substrate. The active region consists of eight 7-nm-wide compressively strained (1\%) InGaAsP Q1.65 wells, alternating with seven 14-nm-wide tensile strained $(0.5 \%)$ InGaAsP Q1.3 barriers, sandwiched between two 10-nm-thick InGaAsP Q1.3 waveguide layers. On top of the InGaAs contact layer, a $0.5-\mu \mathrm{m}$ InP buffer layer and a final $0.1-\mu \mathrm{m}$ InGaAs buffer layer were grown. They were used to protect the contact cap from damage as shallow ion implantation was used to generate quantum-well intermixing (QWI) as in [22]. For this work, we implanted $8 \times 10^{14} \mathrm{P}$ ions at $100-\mathrm{keV}$ energy and $200{ }^{\circ} \mathrm{C}$. With this implantation, energy damage was restricted to the buffer layer, thus, when it was subsequently removed, the surface quality of the contact cap was intact. The wafer was masked with $600 \mathrm{~nm}$ of PECVD $\mathrm{SiO}_{2}$ during implantation in order to protect the gain sections. After implantation, the wafer was rapidly thermally processed at $650{ }^{\circ} \mathrm{C}$ for $90 \mathrm{~s}$, which created a 35-nm blue-shift of the bandgap in the phase and modulator sections. Part of the wafer was then processed into PIN devices, and photocurrent spectra were measured to extract the spectral dependence of the material absorption as a function of reverse bias, as shown in Fig. 4. This measurement was used to calculate the effect of QCSE on refractive index by using the Kramers-Kronig relation (Fig. 5). This showed that the refractive index for the wells changes by 0.17 with $2-\mathrm{V}$ reverse bias at a wavelength of $1560 \mathrm{~nm}$ (Fig. 5). Such an index 


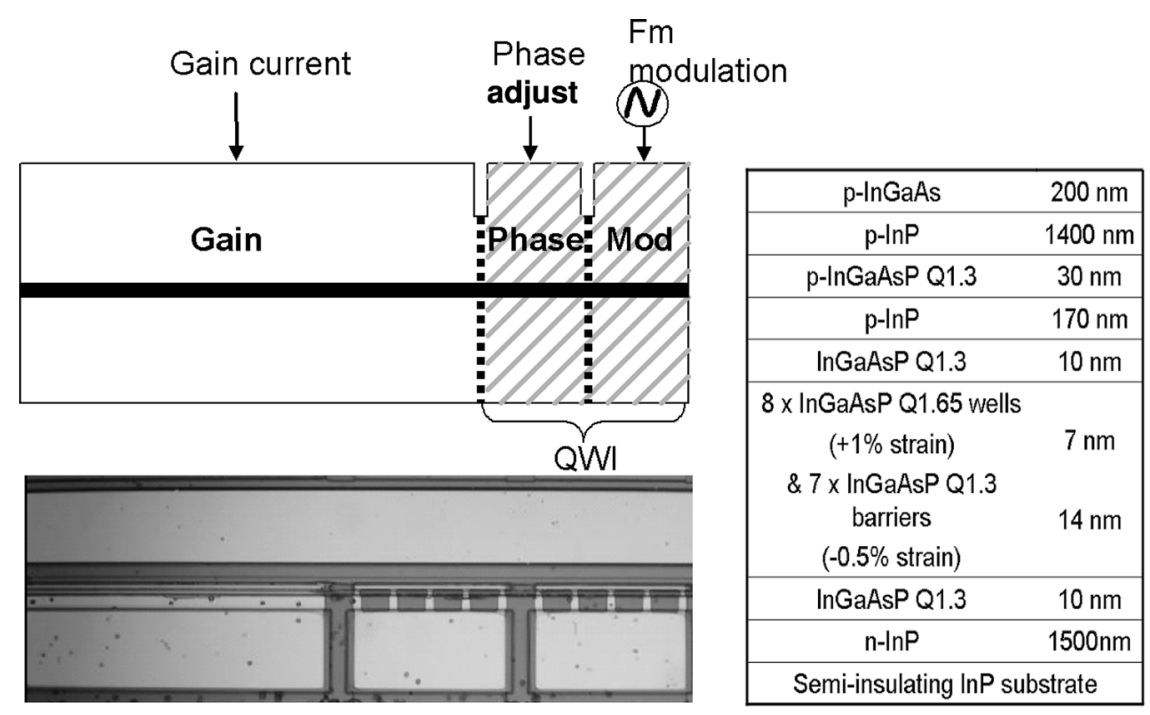

(a)

(b)

Fig. 3. Schematic and optical micrograph of (a) the three-section laser and (b) epitaxial structure.

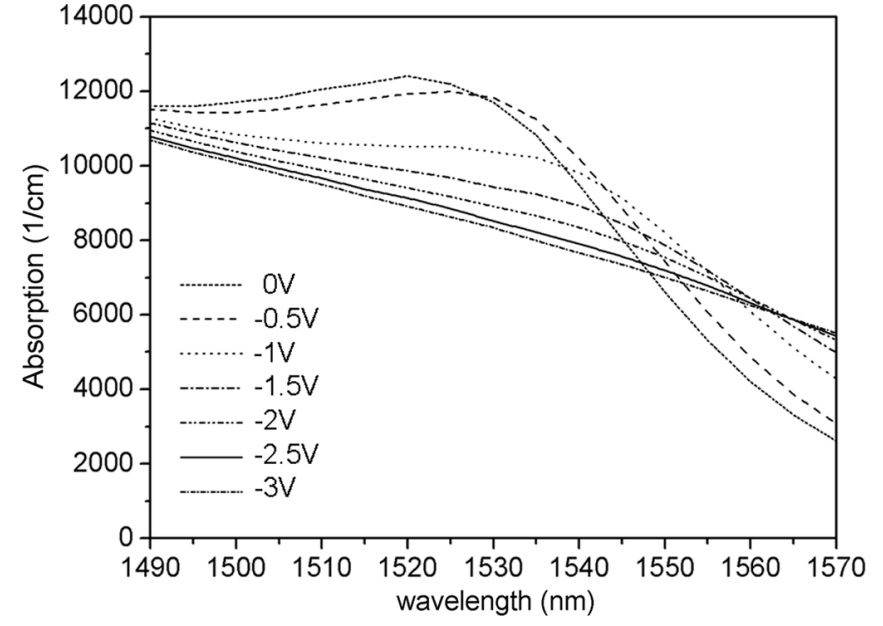

Fig. 4. Absorbtion changes as a function of reverse bias for the propagating mode.

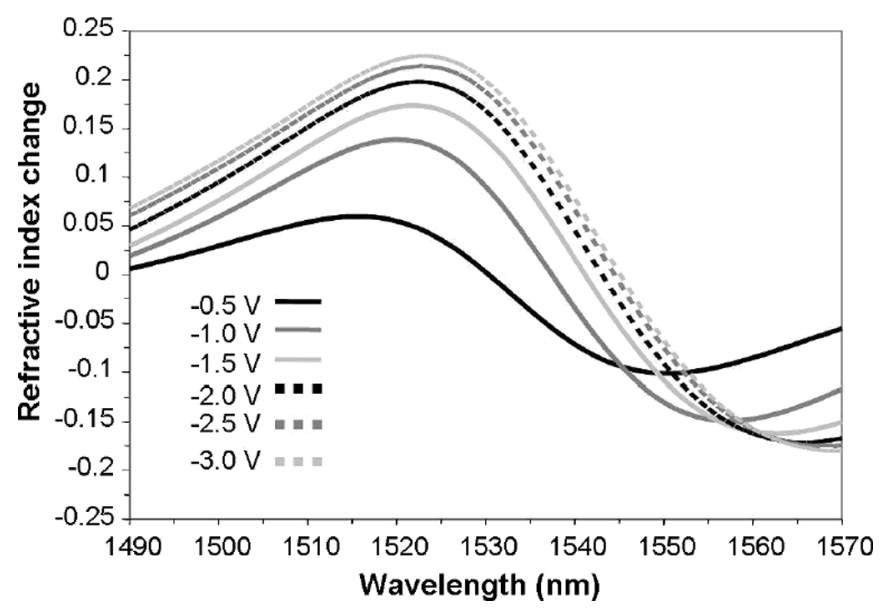

Fig. 5. Refractive index change due to electric field derived from the data of Fig. 4 . The changes quoted are with reference to the zero-field case. change in the 170- $\mu \mathrm{m}$-long modulation section corresponds to a phase change sufficient to create the desired FM laser effect at

$$
\Delta \Phi=\frac{2 \cdot \pi \cdot \Gamma_{\mathrm{MQW}} \cdot \Delta n \cdot L_{m}}{\lambda}=10 \mathrm{rad}
$$

where $\Delta n$ is the refractive index change of the MQW, $\Gamma_{\mathrm{MQW}}$ is the confinement factor of the guided optical mode within the wells, $L_{m}$ is the length of the modulation section, and $\lambda$ is the operating wavelength.

The fabricated laser was a ridge waveguide design with oxidebridged contacts in order to reduce the capacitance of the modulation section (shown in Fig. 3). The different sections were separated by an isolation trench through the highly doped top layers. The oxide-bridged contact for the modulation section allowed a $380-\mathrm{fF}$ measured capacitance and a $20-\Omega$ series resistance resulting in a maximum $-3-\mathrm{dB}$ modulation bandwidth of $21 \mathrm{GHz}$. As both phase section and modulation section were intermixed, the laser threshold remained relatively low at $80 \mathrm{~mA}$ for a $1.88-\mathrm{mm}$-long device. The laser was operated at 200-mA bias current, giving a total output of $2 \mathrm{~mW}(1 \mathrm{~mW}$ coupled into a single-mode fiber).

The isolation trenches induce a small reflection $(\sim 4 \%)$ of the propagating mode creating subcavity effects. If one considers a trench through the top $1.6 \mu \mathrm{m}$ of the structure and a two-section device, a simulation of the coupled Fabry-Perot cavities created will lead to the calculated transmission function shown in Fig. 6. As one can see, the transmissions spectrum shows regular dips (spaced by $2.1 \mathrm{~nm}$ corresponding to a $170-\mu \mathrm{m}$ subcavity) which will strongly affect the output spectrum of the laser. It will offer the advantage of limiting the number of modes operating in the cavity, thus the laser could potentially have single-mode freerunning operation. However, it will also limit the number of comb lines generated as they will be confined to the spectral zones of higher transmission. 


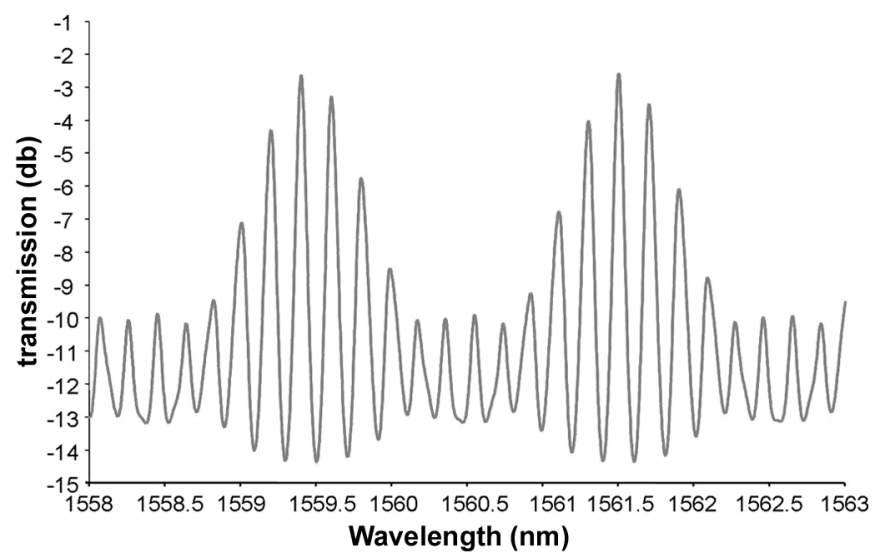

Fig. 6. Transmission of the multiple Fabry-Perot cavity.

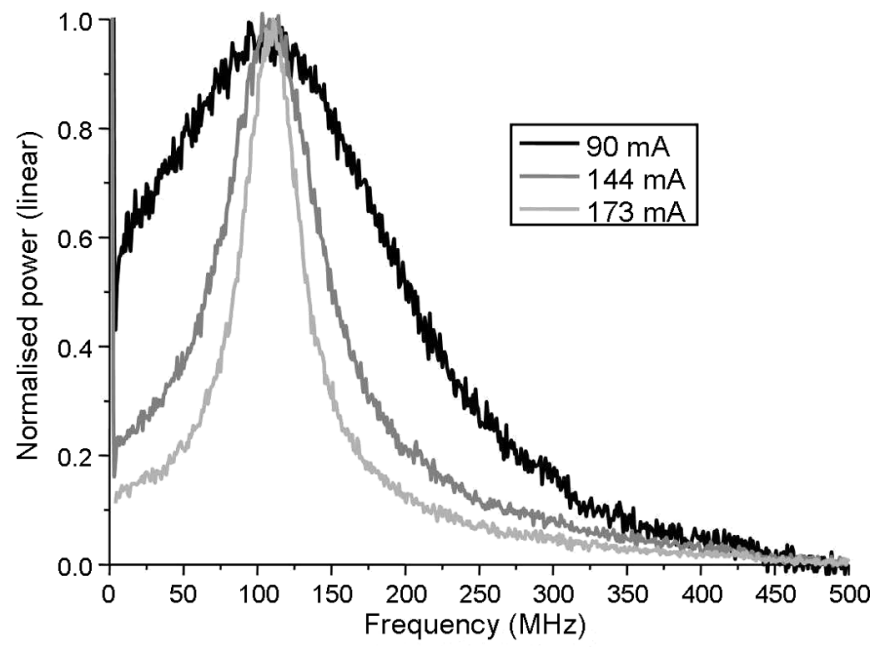

Fig. 7. Free-running laser linewidth for current settings from $90 \mathrm{~mA}$ (just above threshold) to $173 \mathrm{~mA}$ (just below multimode operation).

\section{EXPERIMENTS AND RESULTS}

Note that, for all experimental measurements, the laser output was collected using a lensed fiber with antireflection coating which was spliced to an optical isolator in order to eliminate the unwanted effects on laser operation due to external optical feedback.

\section{A. Free-Running Laser}

In order to optimize the comb generator operation prior to applying modulation, the laser gain current was adjusted to give optimum free-running operation, where the linewidth is at its minimum and the laser operates in a single-mode regime. In order to measure the linewidth of the laser mode, a self-heterodyne system was used [23]. The delay was $5 \mathrm{~km}$, offering a measurement resolution of $40 \mathrm{kHz}$, which should be sufficient as a typical semiconductor laser will have a linewidth of the order of a few megahertz. The linewidth was measured as a function of the gain section current, as shown in Fig. 7. At low bias current, the laser shows the expected linewidth decrease with increased current. However, at a certain bias current (174 mA), the linewidth increases sharply to $200 \mathrm{MHz}$. This was found to be the bias current where the laser starts to operate in a multimode regime. The operation point was therefore chosen to be

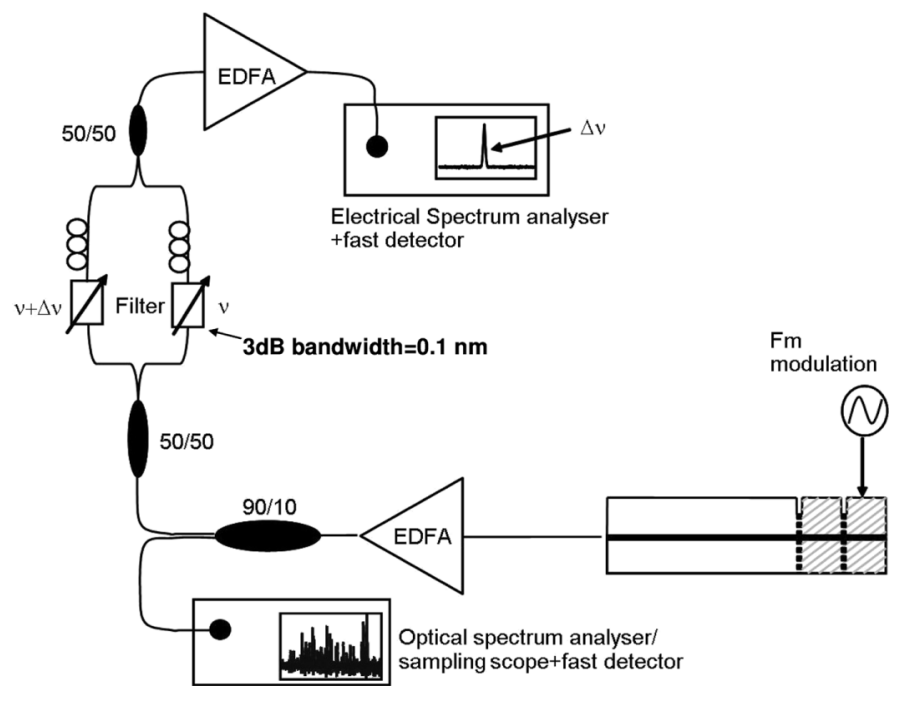

Fig. 8. Experimental system.

just below this current. At this bias, the laser had an output power of $1.2 \mathrm{~mW}(0.6 \mathrm{~mW}$ coupled into a single-mode fiber, with an operating temperature of $20^{\circ} \mathrm{C}$ ) with a linewidth of $25 \mathrm{MHz}$. Note that the measured width on the RF spectrum analyzer is twice the actual linewidth of the laser [23].

\section{B. Modulated Laser}

Fig. 8 shows the different systems used to characterize the FM laser. The output spectrum of the laser was assessed with an optical spectrum analyzer. Finer spectral studies were also done with the same self-heterodyne system used previously on the free-running laser in order to assess whether each comb line was retaining the spectral purity of the free-running laser. The laser output power stability was also studied using a fast photodetector (50-GHz bandwidth) and a sampling oscilloscope which was triggered using a reference output from the frequency generator. This allowed residual intensity modulation on the laser output to be studied. As can be seen from Fig. 4, the absorption of the modulation section will also change with the voltage, thus inducing intensity modulation. Monitoring intensity modulation also enables the transition to mode-locked operation to be observed [21] when the modulation frequency is exactly equal to the axial mode spacing. The stability of the line spacing and its relation to the modulation source frequency were assessed by heterodyning two adjacent lines and sending the resulting signal to the fast photodetector which was connected to a spectrum analyzer. Note that the filters were placed at the output of the erbium-doped fiber amplifiers (EDFAs) to reduce the amplified spontaneous emission (ASE) level in the resulting spectrum. The phase noise as a function of the frequency offset was then extracted and compared with the modulation source phase noise.

Fig. 9 shows the output spectrum of the laser with and without 24.4-GHz modulation. The modulation amplitude was chosen to be $2 \mathrm{~V}$ peak-to-peak signal with $2-\mathrm{V}$ negative bias, where the output spectrum was showing the highest number of comb lines created. With no modulation, lasing was on a single longitudinal mode. With modulation at frequencies much lower than the axial 

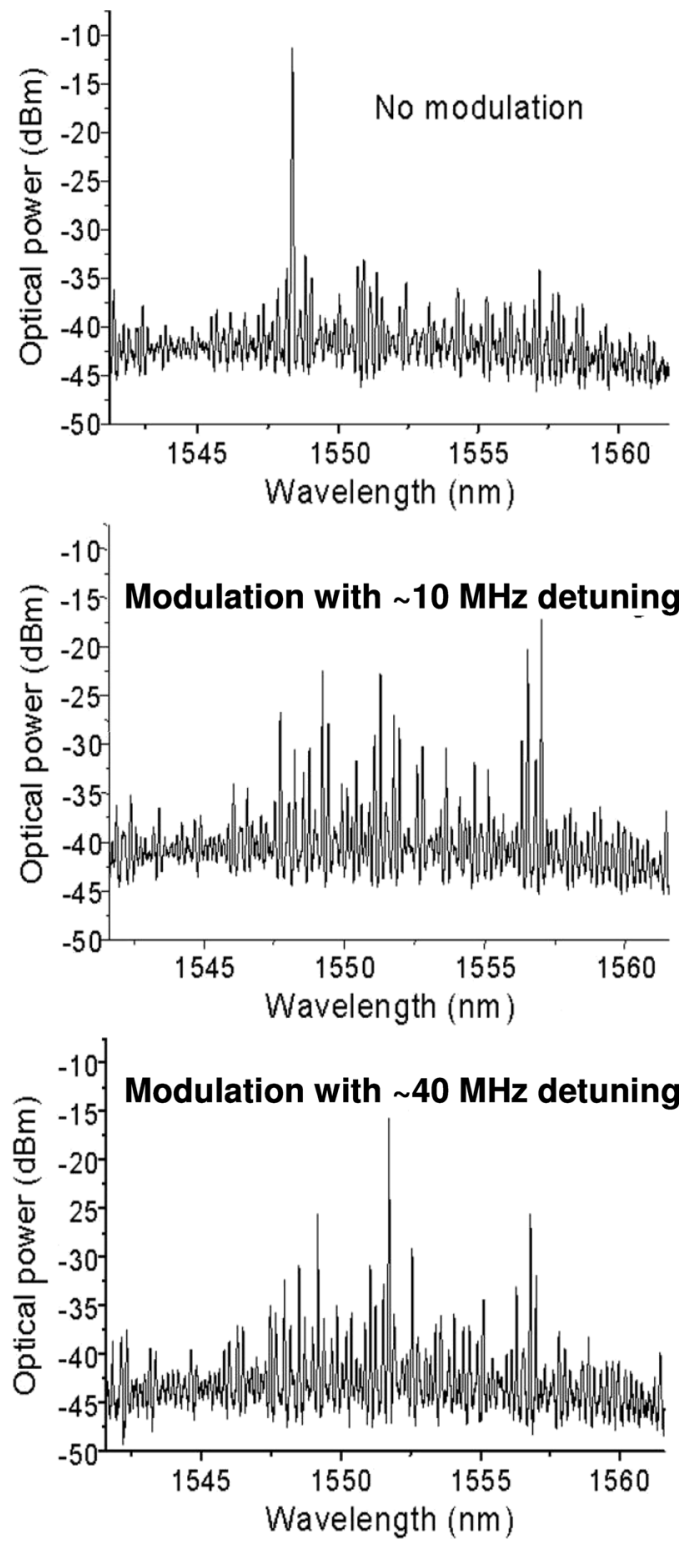

Fig. 9. Spectrums with and without modulation.

frequency ( $>1 \mathrm{GHz}$ detuning using the frequency source), the laser spectrum only showed the peak and a pair of side lines. With modulation closer to the axial frequency, the spectrum shows a number of lines appearing across the spectrum. When the phase was changed in order to detune the laser axial frequency further from the modulation frequency, the number of lines diminished and was closer to the original peak frequency as expected for an FM laser. Note that the spectrum is not symmetric as for the ideal example given previously. This is mainly due to the strong absorption on the short wavelength side of the spectrum, as, for this laser, the free-running peak wavelength was in the short-wavelength side of the gain spectrum. Other lasers operating with a single free-running peak in the center of the gain spectrum operated with a more symmetric comb spectrum, though the number of lines was smaller. This absorption is unavoidable as the intermixing was chosen to be the best balance between low absorption and strong index modulation. This

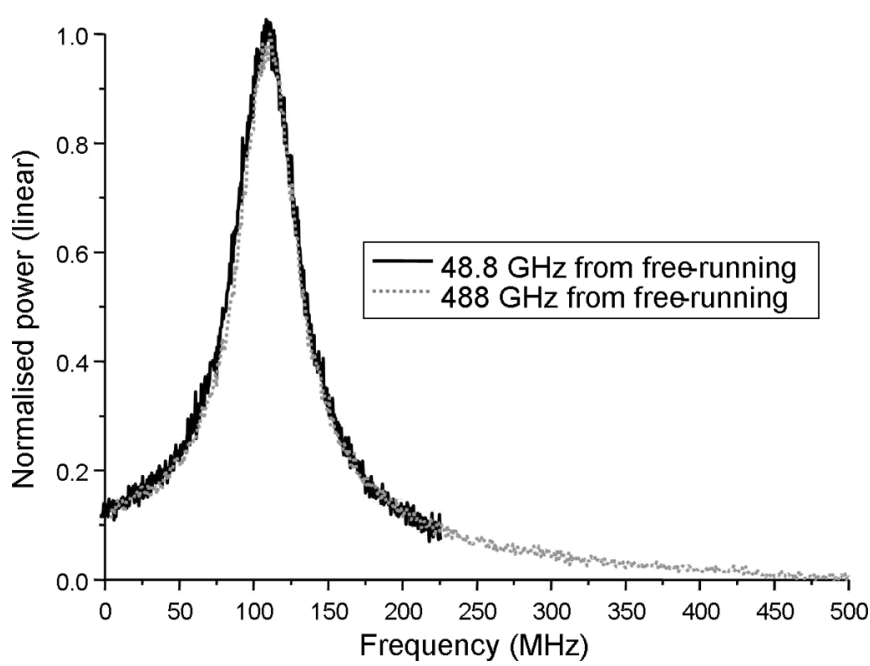

Fig. 10. Comb-lines linewidth measurement.

therefore limits the total span of the comb as it reduces the gain bandwidth. Furthermore, we believe that the trenches used to isolate the different sections, which create a system of coupled Fabry-Perot subcavities, are responsible for the lack of lines created at certain points of the spectrum (Fig. 9). The simulation result, shown in Fig. 6 (for a simpler two-section cavity), indicates that such a cavity design induces higher losses in the cavity at frequency spacing determined by the length of the modulation section. Though the real cavity was not simulated, it is reasonable to assume that a similar effect of zones of higher losses should occur while the periodicity should not be as obvious.

To assess the quality of the created comb lines, their linewidth was measured with the self-heterodyne system described previously, each line being extracted using a $0.1-\mathrm{nm}-3-\mathrm{dB}$ bandwidth filter $(13 \mathrm{GHz})$. Fig. 10 shows the linewidth of different comb lines at a given offset from the original free-running laser frequency. As expected, the comb-line linewidth remains the same as the linewidth of the free-running laser for all comb lines. The linewidth was at its optimum for this laser, i.e., $25 \mathrm{MHz}$.

As a laser operating in the FM regime should show no intensity modulation, to confirm that the device was operating as an FM laser, the stability of its output power was measured with a fast photodetector both on a spectrum analyzer and on a sampling oscilloscope. Fig. 11 shows the result obtained with the sampling oscilloscope at different points of operation. As one can see, at most operation points, the output had almost no intensity modulation. However, up to $20 \%$ intensity modulation appears when the modulation frequency is close to the axial frequency. This was expected as the QCSE only slightly changes the absorption of the modulation section. The spectrum analyzer did also show a weak peak at $24.4 \mathrm{GHz}$ when the modulation frequency was close to the cavity axial frequency, which is in agreement with the previous measurement. In theory, the laser should transition to FM mode-locked operation when the modulation frequency is exactly equal to the cavity axial frequency. When measured at this frequency the laser showed the same behavior as with a small detuning (around $10 \mathrm{kHz}$ ), but no mode-locked regime was observed. It is likely that, in this 

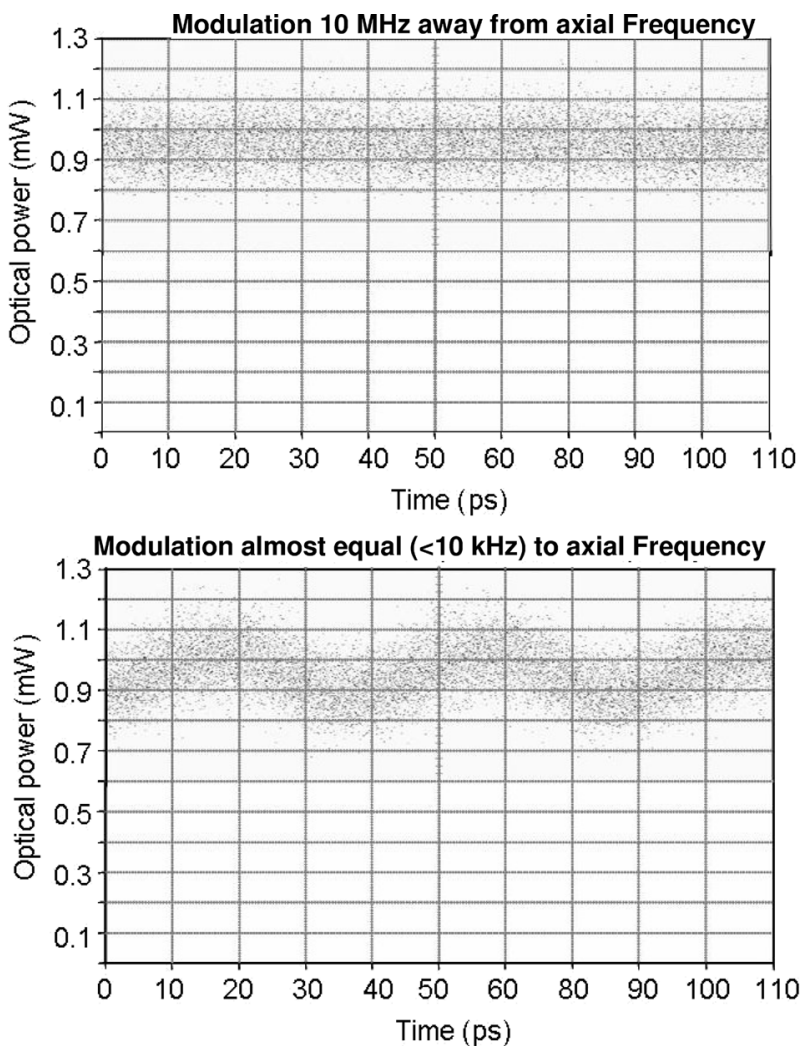

Fig. 11. Amplitude modulation measurement at different modulation points.

device, the intracavity reflections combined with strong cavity dispersion prevented operation in the mode-locked regime.

This experiment was repeated with individual filtered comb lines in order to assess their intensity stability, and no differences were seen in the measurements compared with the measured intensity modulation of the full output of the laser.

In order to confirm FM operation, the comb-line spacing should be measured and its relation to the modulation frequency demonstrated. For this, we used the heterodyne system described previously with $0.1-\mathrm{nm}(13-\mathrm{GHz})$ bandwidth filters on each branch of the system. This also offers the advantage of assessing the relative frequency stability of the line spacing.

Fig. 12 shows the heterodyne result for one given pair of lines. For this measurement, the spectrum analyzer span was $100 \mathrm{kHz}$ and the resolution bandwidth was $1 \mathrm{kHz}$. The line was at the exact frequency of the modulation source and had the same spectral purity, as expected. Thus, as the comb lines were spaced by the modulation frequency, we could definitely conclude that the device was operating as an FM laser. Phase-noise measurements were also made on the heterodyne signal to compare it with the modulation source (Fig. 13). The phase-noise spectral density was obtained (estimated) from the RF power spectrum adjacent to the heterodyne signal (or the modulation source signal) by normalizing to the peak power in the signal, on the assumption that the phase noise dominates over amplitude noise [24]. The noise floor of the spectrum analyser was around $-125 \mathrm{dBm} / \mathrm{Hz}$ (check powers) or less for all frequency offsets, while the peak signal powers were around $10 \mathrm{dBm}$ for the modulation source and $-10 \mathrm{dBm}$ for the heterodyne signal, giving noise floors for the phase noise measurements of around

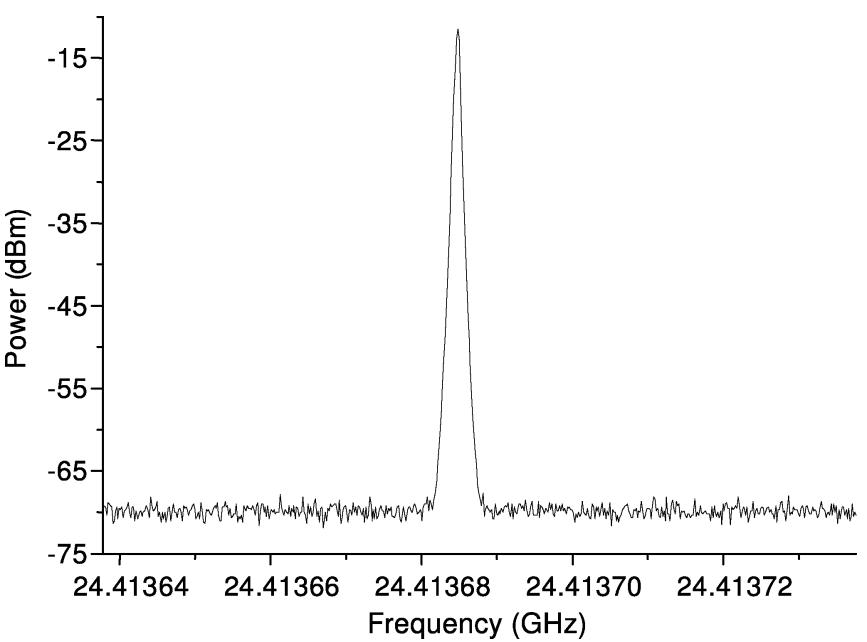

Fig. 12. Electrical spectrum of the heterodyne signal created from two adjacent lines of the comb generator.

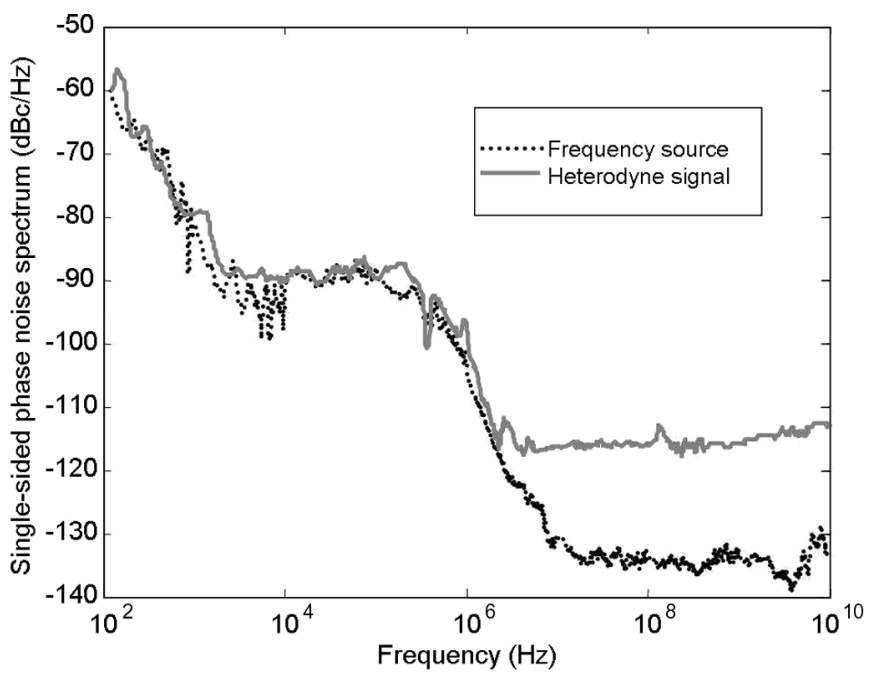

Fig. 13. Phase-noise measurement of the heterodyne signal from two adjacent comb lines.

-135 and $-115 \mathrm{dBc} / \mathrm{Hz}$, respectively. As can be seen, up to a frequency offset of about $2 \mathrm{MHz}$, the phase noise is the same for the source and the heterodyne signal; above this frequency offset, the noise floor of the spectrum analyzer is reached and no comparison can be made. However, this shows that phase noise of $-82 \mathrm{dBc} / \mathrm{Hz}$ at $1-\mathrm{kHz}$ offset and $-108 \mathrm{dBc} / \mathrm{Hz}$ at $1-\mathrm{MHz}$ offset can be achieved.

An important issue for the use of this laser as a frequency reference is its absolute frequency stability. Typically, a semiconductor laser will show an absolute frequency drift due to the current source instability and thermal drift. The FM laser free-running peak wavelength drift was measured over several hours by heterodyning the laser output with the output of a stabilized DFB laser (on a molecular resonance-acetylene-absolute frequency stability $<100 \mathrm{kHz}$ ) and observing it on an RF spectrum analyzer. It was found to be about $200 \mathrm{MHz}$ with an approximately linear drift for the duration of the measurement. This indicates that the drift is mainly due to the control electronics. Note that this corresponds to a change of the axial frequency of about $100 \mathrm{kHz}$. This is small enough to not affect 
the FM operation of the laser and confirm the fact that we did not see any change in the comb spectrum over a similarly long operation of the FM laser. In order to obtain a higher absolute frequency stability, the laser will need to be locked on an atomic or molecular resonance [25].

\section{CONCLUSION}

We have shown the use of a combination of different fabrication techniques in the quaternary/quaternary InGaAsP-InP material to realize a monolithic FM laser comb generator. First, the bandgap of part of the wafer was blue shifted using QWI by shallow ion implant and rapid thermal processing (RTP). The 35-nm bandgap shift obtained allowed low signal absorption in the phase and modulation sections of the device. Second, the fabrication of the device was made using oxide-bridge techniques to reduce the capacitance of the modulation section to $380 \mathrm{fF}$. This allowed for an efficient reverse bias modulation using QCSE at $24.4 \mathrm{GHz}$, which was close to the $3-\mathrm{dB}$ bandwidth of the modulation section $(21 \mathrm{GHz})$. The optical frequency comb generator could potentially offer $24.4-\mathrm{GHz}$ spaced lines over a spectral width of $15 \mathrm{~nm}(\sim 2 \mathrm{THz})$. However, the complexity of the cavity, dispersion and the losses induced by the material were limiting the number of lines created of the potential span. The created laser lines were exactly spaced by the modulation frequency and the residual intensity modulation was less than $20 \%$. Each line had the same linewidth of $25 \mathrm{MHz}$ as the nonmodulated laser. When heterodyned, two adjacent lines gave a high-purity $(-82 \mathrm{dBc} / \mathrm{Hz}$ st $1-\mathrm{kHz}$ offset) frequency with phase noise limited by the frequency source. Another limitation for the source is that its absolute frequency stability is limited. A 200-MHz drift measured over a period of several hours was mainly due to the control electronics. We believe that this could be solved by locking the laser on an atomic or molecular resonance. We have isolated two main issues to tackle first in order to improve the performances of the monolithic comb generator. They are the multiple-cavity structure and the span of the comb. For the first one, we plan to use ion implantation as the section electrical isolation mechanism [26] in order to reduce the level of residual reflection. For the second issue, the use of distributed bandgap material will be investigated in order to increase the gain bandwidth [27] of the laser as well as, potentially, the modulation depth [28]. In that case, we believe that such monolithic comb generators could provide a compact alternative to fiber-loop techniques with larger comb-line output powers than passive phase-modulator techniques.

\section{REFERENCES}

[1] O. P. Gough, C. F. C. Silva, S. Bennett, and A. J. Seeds, "Zero frequency error DWDM channel synthesis using optical injection-locked comb line selection," Electron. Lett., vol. 35, pp. 2050-2052, 1999.

[2] S. Fukushima, C. F. C. Silva, Y. Muramoto, and A. J. Seeds, "10 to $110 \mathrm{GHz}$ tunable opto-electronic frequency synthesis using optical frequency comb generator and uni-travelling-carrier photodiode," Electron. Lett., vol. 37, pp. 780-781, 2001.

[3] A. J. Seeds, C. C. Renaud, M. Pantouvaki, M. Robertson, I. Lealman, D. Rogers, R. Firth, P. J. Cannard, R. Moore, and R. Gwilliam, "Photonic synthesis of THz signals," in Proc. Eur. Conf. Microw., Manchester, U.K., 2006, pp. 1784-1787, (invited paper).

[4] A. J. Seeds, C. C. Renaud, M. Pantouvaki, M. Robertson, D. Rogers, and P. J. Cannard, "Efficient photonic THz generation," in Proc. Asia Pacific MWP, Kobe, Japan, 2006, pp. G4_1-G4_24, (Invited paper).
[5] C. W. Oates, M. Stephens, and L. Hollberg, "All-diode-laser optical frequency reference using laser-trapped calcium," in Proc. Annu. IEEE Int. Frequency Control Symp., 1997, pp. 219-224.

[6] Y. C. Chunga and R. M. Derosier, "Frequency-locking of $1.5-\mu \mathrm{m} \mathrm{In-}$ GaAsP lasers to an atomic krypton line without dithering the laser frequency," IEEE Photon. Technol. Lett., vol. 2, no. 6, pp. 435-437, Jun. 1990.

[7] T. Ikegami, S. Sudo, and Y. Sakai, Frequency Stabilization of Semiconductor Laser Diodes. Norwell, MA: Artech House, 1995.

[8] J. Lucero, Y. C. Chung, and R. W. Tkach, "Survey of atomic transitions for absolute frequency locking of lasers for lightwave systems," IEEE Photon. Technol. Lett., vol. 3, no. 5, pp. 484-486, May 1991.

[9] R. Boucher, B. Villeneuve, M. Bretona, and M. Tetu, "Calibrated Fabry-Perot etalon as an absolute frequency reference for OFDM communications," IEEE Photon. Technol. Lett., vol. 4, no. 7, pp. 801-804, Jul. 1992.

[10] E. T. Peng, S. F. Bed, and C. B. Su, "Frequency stabilization of a traveling-wave semiconductor ring laser using a fiber resonator as a frequency reference," IEEE Photon. Technol. Lett., vol. 6, no. 3, pp. 334-337, Mar. 1994.

[11] N. Shimosaka, K. Kaede, M. Fujiwara, S. Yamazaki, S. Murata, and M. Nishio, "Frequency separation locking and synchronization for FDM optical sources using widely frequency tunable laser diodes," IEEE J. Sel. Areas Commun., vol. 8, no. 6, pp. 1078-1086, Aug. 1990.

[12] M. Kourogi, K. Nakagawa, and M. Ohtsu, "Wide-span optical frequency comb generator for accurate optical frequency difference measurement," IEEE J. Quantum Electron, vol. 29, no. 10, pp. 2693-2701, Oct. 1993.

[13] K. Imai, M. Kourogi, and M. Ohtsu, "30-THz span optical frequency comb generation by self-phase modulation in an optical fiber," IEEE J. Quantum Electron, vol. 34, no. 1, pp. 54-60, Jan. 1998.

[14] S. Bennet, B. Cai, E. Burr, O. Gough, and A. J. Seeds, "Terahertz, zero frequency error, tunable optical comb generator for DWDM applications," presented at the Conf. Optical Fiber Commun., San Diego, CA, 1999, Paper WM5, unpublished.

[15] P. Shen, N. J. Gomes, P. A. Davies, W. P. Shillue, P. G. Huggard, and B. N. Ellison, "Fibre ring based optical frequency comb generator with comb line spacing tuneability," presented at the LEOS Summer Top. Meet., San Diego, CA, 2005, Paper MB 2.2.

[16] M. Teshima, M. Koga, and K. Sato, "Accurate frequency control of a mode-locked laser diode by reference-light injection," Opt. Lett., vol. 22, pp. 126-128, 1997.

[17] O. J. Koninb, A. C. Labrujere, C. M. de Blok, and J. P. Bekooij, "Reference frequency-comb for multi-channel stabilization by mode-locking of a semiconductor laser," in Proc. Eur. Conf. Opt. Commun., 1990, pp. 539-542.

[18] S. Gee, F. Quinlan, S. Ozharar, P. J. Delfyett, J. J. Plant, and P. W. Juodawlkis, "Optical frequency comb generation from modelocked diode lasers-Techniques and applications," presented at the LEOS Summer Top. Meet., San Diego, CA, 2005, Paper MB 2.3, unpublished.

[19] D. J. Kuizenga and A. Siegman, "FM-laser operation of the Nd:YAG laser," IEEE J. Quantum Electron., vol. QE-6, no. 11, pp. 673-677, Nov. 1970.

[20] T. Tuetken, G. Frankowsky, A. Hangleiter, V. Haerle, K. Streubel, and F. Scholz, "Quantum confined stark effect in InGaAs/InP and InGaAs/ InGaAsP multi quantum well structures," Superlattices Microstruct., vol. 7, pp. 309-313, 1990.

[21] D. J. Kuizenga and A. E. Siegman, "FM and AM mode locking of the homogeneous laser-1, 2," IEEE J. Quantum Electron., vol. QE-6, no. 10, pp. 694-715, Oct. 1970.

[22] E. J. Skogen, J. W. Raring, J. S. Barton, L. A. Johansson, and L. A. Coldren, "Postgrowth control of the quantum-well band edge for the monolithic integration of widely tunable lasers and electroabsorption modulators," IEEE J. Sel. Topics Quantum Electron., vol. 9, no. 5, pp. 1183-1190, Sep./Oct. 2003.

[23] L. Richter, H. Mandelberg, M. Kruger, and P. McGrath, "Linewidth determination from self-heterodyne measurements with subcoherence delay times," IEEE J. Quantum Electron., vol. QE-22, no. 11, pp. 2070-2074, Nov. 1986.

[24] F. M. Gardner, Phaselock Techniques, 3rd ed. New York: Wiley Interscience Edition, 2005.

[25] C. R. Schwarze and J. H. Rentz, "Frequency stabilized distributed feedback laser diode system at $1.323 \mu \mathrm{m}$ using the modulated Zeeman effect," Rev. Sci. Instrum., vol. 70, pp. 3828-3831, 1999.

[26] S. F. Kong, M. Pantouvaki, C. P. Liu, A. J. Seeds, P. Too, S. Ahmed, R. Gwilliam, and J. S. Roberts, "Electrical isolation of MQW InGaAsP/InP structures by $\mathrm{MeV}$ iron ion implantation for vertical PIN modulators and photodiodes," in Proc. Int. Conf. Indium Phosphide and Related Mater., 2004, pp. 362-365. 
[27] C.-F. Lin, Y.-S. Su, and B.-R. Wu, "Extremely broadband tunable semiconductor lasers for optical communication," in Proc. Conf. Lasers Electro-Opt., 2001, pp. 237-2388.

[28] M. Silver, M. P. D. Greene, and A. R. Adams, "Improved polarization-insensitive long-wavelength quantum-confined-Stark-effect modulators based on multiple-strain-stepped quantum wells," in Proc. Conf. Lasers Electro-Opt., 1995, pp. 289-290.

Cyril C. Renaud was born in Paris, France, in 1973. He received the degree of engineering from the Ecole Supérieure d'Optique, Orsay, France, the Diplôme d'Etudes Approfondies (D.E.A.) in optics and photonics from the University Paris XI, Orsay, France, in 1996, and the Ph.D. degree from the University of Southampton, Southampton, U.K., in 2001.

He spent one year as a Project Engineer with Sfim-ODS, where he was involved with the development of microchips lasers. He then joined Optoelectronics Research Centre, University of Southampton, where he was involved with diode-pumped high-power ytterbium-doped fiber lasers, with particular interest on $Q$-switched systems and $980-\mathrm{nm}$ generation. He is currently working on optoelectronic devices and systems with the Ultra-Fast Photonics Group, University College London, London, U.K. His current research includes uncooled WDM sources, agile tunable laser diode and monolithic optical frequency comb generator using quantum-confined Stark effect, high-frequency photodetectors (UTC, traveling wave), and optical frequency generation systems in the optical and millimeter-wave domains (DWDM, THZ).

Marianna Pantouvaki received the degree in physics from the University of Athens, Athens, Greece, in 1998, and the M.Sc. degree in telecommunications and the Ph.D. degree in electronic engineering from University College London, London, U.K., in 1999 and 2004, respectively. Her doctoral work concerned multiple-quantum-well saturable absorbers with picosecond recovery times for high-bit-rate optical communications.

She is currently working on semiconductor laser amplifiers integrated with saturable absorbers, quantum-confined Stark effect (QCSE) tunable lasers, and optical comb generators with the Ultra-Fast Photonics Group, University College London.

Sylvie Grégoire received a degree of engineering from the Conservatoire National des Arts et Metier (CNAM), Paris, France, in 2006.

She has been with the Department of Optics, Ecole Nationale Superieure de Telecom Bretagne (ENST Bretagne), Brest, France, since 2002. Her work is mainly centered on liquid crystal and lithography. She spent nine months (2005-2006) as a Visiting Research Assistant with the Photonics Group, University College London, London, U.K., to study monolithic MQW InP-InGaAsPbased optical frequency comb generators.

Ian Lealman (M'01) received the degree in physics from the University of Southampton, Southampton, U.K., in 1985 and the Ph.D. degree from the University of Essex, Essex, U.K., in 1999. His doctoral work focused on mode-expanded lasers.

He subsequently joined BT Labs, Ispwich, U.K., where he was involved with the development of BH laser technology and was heavily involved in transferring it into production at BT\&D. In 1990, he returned to BT Labs to continue research into a wide range of optoelectronic devices, including high-speed semiconductor lasers, monolithic widely tunable lasers, and mode-expanded lasers and semiconductor optical amplifiers. In 1996, he also became the Project Manager for BT's photonics research. From 2000 to 2003, he was Department Manager for active design and fabrication at the Corning Research Centre in the U.K. He joined the Centre for Integrated Photonics (CIP), Ipswich, at its inception in 2003 and is currently Vice President of Device Development as well a Product Manager for CIP's range of semiconductor optical amplifiers. He holds eight U.S. patents and has authored or coauthored over 50 publications in journals and international conference proceedings.

Paul Cannard received the degree in metallurgy and materials science and the Ph.D. degree from University College Cardiff, Cardiff, U.K., in 1985 and 1989, respectively. His doctoral work focused on aluminium nitride and its polytypes by atomic imaging TEM.

He joined the analytical group at British Telecom Research Labs in 1988, where he used SIMS, Auger spectroscopy, TEM, and SEM to study semiconductor and silica fiber-based technologies. From 1996, he ran a VG80 MBE reactor growing electroabsorption modulator material to study the potential use of antimonides as mirrors for InP-based VCSELs. He transferred to Corning Research Centre in 2000 and subsequently joined the Centre for Integrated Photonics, Ipswich, U.K., in 2004 as an Analytical Scientist for epitaxial materials He now works with an Aixtron multiwafer reactor to produce planar epitaxial material for a variety of device programs and with a Thomas Swan atmospheric reactor for overgrowths of buried-heterostructure optoelectronic devices. $\mathrm{He}$ holds one U.S. patent and is a coauthor on over 20 publications in journals and international conference proceedings.

Simon Cole received the degree in materials science from Imperial College, London, U.K., and the Ph.D. degree from the University of Southampton, Southampton, U.K.

He has 15 years' experience in semiconductor materials research. After two years of post-doctoral research at Stanford University and Birmingham University, he joined the MOVPE Growth Group at BT Research Laboratories in 1984, where he played a key role in the development of overgrowth technologies and was part of the team that won the Queen's Award for Technological Achievement in 1993 for successful transfer of MOVPE-grown device technologies to production. After a number of years as a Project Manager in software development at BT Laboratories, he joined Corning Research Centre to develop MOVPE overgrowth and butt-coupling technologies in support of device programs. His recent research interests include selective area growth mechanisms around mesa structures and in situ etching and regrowth. He has authored or coauthored over 20 scientific papers or book articles and a number of patents and has presented work at several international conferences (one invited). He is now with the Centre for Integrated Photonics, Ipswich, U.K.

Ron Moore joined BT in 1987 in the MOVPE growth area, was promoted to Technical Officer in 1990, and was a member of the team that won a Queen's Award for Technological Achievement in 1993. He transferred to Corning in February 2000, with the formation of the Corning Research Centre and was promoted to Scientist grade in 2001. He joined the Centre for Integrated Photonics, Ipswich, U.K., at its inception in 2003 and is currently a Semiconductor Process Engineer responsible for the Centre's multiwafer growth kit.

Russell Gwilliam is the Director of Technology for the Ion Beam Centre, University of Surrey, Guildford Surrey, U.K. For the last 20 years, he has developed advanced processes and equipment for both III- $\mathrm{V}$ and silicon devices and is an active consultant with several international companies. Much of his recent work has been in the area of ion-beam synthesis of novel materials, including silicides in both single crystal and amorphous silicon. He is a coinventor of the silicon light-emission technology on which he founded Si-Light Technologies Ltd. He has published more than 250 papers and is a regular invited speaker at international conferences.

Mr. Gwilliam is a member of both the IEE and IoP and sits on several international conference scientific committee's.

Alwyn J. Seeds (M'81-SM'92-F'97) received the Ph.D. and D.Sc. degrees from the University of London, London, U.K.

From 1980 to 1983, he was a Staff Member with Lincoln Laboratory, Massachusetts Institute of Technology, Cambridge, where he was involved with GaA monolithic millimeter-wave integrated circuits for use in phased-array radar. He returned to England in 1983 to take up a lectureship in telecommunications at Queen Mary College, University of London, moving to University College London in 1986, where he is now Professor of Opto-electronics and Head of the Department of Electronic and Electrical Engineering. He has published over 250 papers on microwave and opto-electronic devices and their systems applications and is presenter of the video "Microwave Opto-electronics" in the IEEE Emerging Technologies series. His current research interests include microwave bandwidth tunable semiconductor lasers, semiconductor optical modulators, mode-locked lasers, optical phase-locked loops, optical frequency synthesis, broadband wireless over fiber access systems, dense WDM networks, $\mathrm{THz}$ photonics, and nonlinear optical devices. He is a cofounder of ZinWave, Inc., a manufacturer of wireless over fiber systems.

Prof. Seeds is Chairman of the Photonics Professional Network of the Institution of Engineering and Technology (U.K.), a Fellow of the Royal Academy of Engineering, and a Member of the Technical Committee on Microwave Photonics of the IEEE Laser and Electro-Optics Society. He has served on the program committees for many international conferences. 